\title{
Health Promotion Challenges in Healthy Lifestyles: Can We Transfer Lessons Learned Across Contexts?
}

\section{Arja R Aro* and Maja Bertram}

Unit for Health Promotion Research, Institute of Public Health, University of Southern Denmark, Niels Bohrs Vej 9, Esbjerg 6700, Denmark

\section{Abstract}

This article argues that multi-level and contextual approach, including evidence-informed approach with needs assessment, is needed for sustainable lifestyle change. Examples are given of older and newer whole of community approaches with health in all policies approach as well as on targeting those at increased risk of diabetes type 2. At their best, these approaches empower local people and creates ustainable community structures and policies for healthy life. The commentary further argues that this kind of needs-based and tailored interventions can be transferred across contexts and cultures. To enable this, we need to pay attention to the intervention context as well intervention process characteristics instead of focusing on the contents of the intervention.

\section{Publication History:}

Received: October 24, 2017

Accepted: December 02, 2017

Published: December 04, 2017

\section{Keywords:}

Healthy lifestyle, Overweight, Obesity, Diabetes, Stakeholders

\section{It Takes more than Knowledge to make Life Style Changes}

The increasing overweight and obesity problems with their health consequences around the world are alarming. The problem is hardly lack of knowledge on the determinants of healthy lifestyle. Most people (in developed countries) know that eating less, eating more vegetables and fruit and less meat and carbohydrates, and being physically active, are the basic ways to avoid weight gain. Why is this knowledge not transferred into practice then?

One central reason is that individual behaviors are highly contextdependent. Human eating habits are not only rational choices; they are influenced by the individuals' own desires and beliefs but also by behaviors and beliefs of those close and important to them and prevalent in their cultural and economic context; they are influenced also by media advertisement, by price and availability of food. In the same way, physical activity does not happen only by individual choice: people might lack time, facilities might be far and expensive, culture or climate might limit at least outdoor activities etc.

The social/ecological model of health illustrates the multiple levels (individual level, family and community level, society level) that are needed for effective and sustainable strategies to tackle public health challenges such as overweight and obesity [1]. Health promotion provides strategies to enable people to increase control over, and to improve, their health, to develop competences for healthy lifestyle in individuals and groups (health education) and to provide the best structures in society to make the healthy choice the easiest choice (health policies).

\section{Examples of Multilevel Health Promotion Interventions}

\section{North Karelia project}

In the past, the North Karelia Project was the model for numerous society and community level programs. It was developed in the early 1970's as an answer to alarming cardiovascular mortality and morbidity in Finland. In 30 years of North Karelia project, which combined country-wide media campaigns, the industry developing new, lighter products, policy changes to favor e.g. low-fat milk by farmers, health care improving e.g. blood pressure control, NGO work, school and work place canteens, was shown $85 \%$ decrease of cardiovascular mortality among males 35- 64 year [2]. A review [3] has shown that most of the interventions copying the 1970's North Karelia project and receiving poorer results, failed to adjust the interventions to their contexts of different time, place and culture.

\section{Seinajoki whole of community example of curbing childhood obesity}

Seinajoki in Finland has implemented a strategy to tackle overweight among children. In 2010 almost 1 in 5 five-year-olds was overweight or obese; six years after the program began, the proportion was halved [4]. The intervention was complex; it aimed at changing eating and physical activity habits via several channels (improving school playgrounds, more physical activity in schools, eliminate sugary snacks, serve healthier lunches, yearly health examinations in schools, parent education on healthy eating). It was multilevel since it focused on making changes at family level (parent education), community level (healthy food, increased activity as part of teaching activities in schools) and structural level (playgrounds, health examinations). Finally, it was intersectoral, since it included participation and action from several of the municipality departments (urban planning, recreation, nutrition, health); thus, following the principle of Health in All Policies [5].

\section{Preventing diabetes among those at increased risk}

Diabetes type 2 is becoming a major health care burden; it is urgent to screen those at increased risk and provide them specially tailored programs. Efficacy trials in controlled conditions have shown that lifestyle interventions can be equal or better than drug trials in both weight loss and diabetes risk reduction [6]. Systematic reviews $[7,8]$ summarizing the evidence of real-world implementation of diabetes prevention interventions, has shown that they can both decrease body weight and diabetes risk factors even with low intervention intensity

*Corresponding Author: Dr. Arja R Aro, Unit for Health Promotion Research, Institute of Public Health, University of Southern Denmark, Niels Bohrs Vej 9, Esbjerg 6700, Denmark; Email: araro@health.sdu.dk

Citation: Aro AR, Bertram M (2017) Health Promotion Challenges in Healthy Lifestyles: Can We Transfer Lessons Learned Across Contexts? Int J Community Fam Med 2: 132. https://doi.org/10.15344/2456-3498/2017/132

Copyright: @ 2017 Aro et al. This is an open-access article distributed under the terms of the Creative Commons Attribution License, which permits unrestricted use, distribution, and reproduction in any medium, provided the original author and source are credited. 
if participation rate is good and interventions last long. The authors [8] concluded that not all the reviewed studies reported intervention characteristics such as penetration, implementation, participation and effectiveness, which would help to transfer the lessons learned to other contexts. A recent major study in Kerala, India [9] shows how a community diabetes Type 2 study can be done based on proper community needs analysis to increase the fit to the context.

\section{Memory Rule to Transfer Good Experiences across Contexts}

When planning complex, context-dependent, multilevel interventions for lifestyles, research evidence can tell us about the size of the problem, potentially effective interventions and implementation strategies. However, to make a sustainable change in any setting, in addition to research evidence, it is important also to consider knowledge from the involved stakeholders (e.g. community stakeholders) and the values, norms and wishes of the target population(e.g. school children, teachers, families) as well as social, cultural, legal and economic conditions. For all lifestyle interventions, needs assessment in the local settings is a solid starting point that can ensure inclusion of knowledge and involvement of the stakeholders and target groups. For recent examples on how to integrate research knowledge to real world policy making in physical activity, please see Aro et al. 2015 [10], Bertram et al. 2016 [11], www.repopa.eu/ publications.

\section{Discussion and Conclusion}

Both research and experience from different contexts show that multi-level and contextual interventions based on health in all policies thinking with needs assessment, are needed for sustainable lifestyle change. To expand the transferable know-how, we need to pay attention to the intervention contextual and process characteristics instead of focusing on the intervention contents. We also need to report transparently why and how we ran the intervention and what worked well for whom; shortly, we need proper process evaluation when carrying out lifestyle interventions in any context.

\section{Competing Interests}

The authors declare that they have has no competing interests.

\section{References}

1. Göran D, Whitehead M (1991) Policies and strategies to promote equity in health. Institute for Future Studies: Stockholm.

2. Puska P, Vartiainen E, Nissinen A, Laatikainen T, Jousilahti P, et al. (2016) Background, Principles, Implementation, and General Experiences of the North Karelia Project. Glob Heart 11: 173-178.

3. McLaren L, Ghali LM, Lorenzetti D, Rock M (2007) Out of context? Translating evidence from the North Karelia project over place and time. Health Educ Res 22: 414-424.

4. WHO (2015) Finland curbs childhood obesity by integrating health in all policies.

5. Leppo K, Ollila E, Pẽ na S, Wismar M, Cook S, et al. (2013) Health in All Policies. Seizing opportunities, implementing policies. Ministry of Socia Affairs and Health Finland.

6. Yoon U, Kwok LL, Magkidis A (2013) Efficacy of lifestyle interventions in reducing diabetes incidence in patients with impaired glucose tolerance: a systematic review of randomized controlled trials. Metabolism 62: 303-314.

7. Johnson M, Jones R, Freeman C, Woods HB, Gillett M, et al. (2013) Can diabetes prevention programmes be translated effectively into real-world settings and still deliver improved outcomes? A synthesis of evidence. Diabetic Medicine 30: 3-15.

Int J Community Fam Med

ISSN: $2456-3498$
8. Aziz Z, Absetz P, Oldroyd J, Pronk NP, Oldenburg B et al. (2015) A systematic review of real-world diabetes prevention programs: learnings from the last 15 years. Implement Sci 10: 172.

9. Daivadanam $M$, Absetz $P$, Sathish T, Thankappan KR, Fisher EB et al. (2013) Lifestyle change in Kerala, India: needs assessment and planning for a community-based diabetes prevention trial. BMC Public Health 13: 95.

10. Aro AR, Bertram M, Hämäläinen RM, Van De Goor I, Skovgaard T, et al. (2016) Integrating research evidence and physical activity policy makingREPOPA project. Health Promotion International 31: 430-439.

11. Maja B, Christina R, Natasa L, Malene T, Thomas S, et al. (2016) Planning Locally Tailored Interventions on Evidence Informed Policy Making - Needs Assessment, Design and Methods. Health Systems And Policy Research 3: 1-10. 